\title{
Parametric Model Approach for Rapid SAR Measurements
}

\author{
O. Merckel $^{1}$, J.-Ch. Bolomey ${ }^{1}$, G. Fleury ${ }^{2}$ \\ ${ }^{1}$ Department of Electromagnetic Research, \\ SUPELEC, \\ 3 rue Joliot-Curie, -91192 Gif-sur-Yvette Cedex, France \\ Phone: +33 (0)1 69851565, Fax: +33 (0)1 69851765, E-mail: olivier.merckel@ supelec.fr \\ ${ }^{2}$ Department of Measurement \\ SUPELEC \\ 3 rue Joliot-Curie, -91192 Gif-sur-Yvette Cedex, France \\ Phone: +33169851425, Fax: +33 169851429 , E-mail: Gilles.Fleury@supelec.fr.
}

\begin{abstract}
Specifc Absorption Rate (SAR) designates the electromagnetic power density deposited per unit mass of biological tissues. This paper presents a new approach where SAR calculation for mobile phones is based on a parametric reconstruction of the E-feld distribution in the phantom to assess rapid SAR measurements, by means of an ellipsoidal model. The estimation of its parameters is achieved by two ways: using a reduced number of real data points exclusively, and using a combination of real data points and E-feld extrapolation with a given expansion model.
\end{abstract}

Keywords - Dosimetry, Rapid SAR, Phaseless Near-Field technique, Parametric reconstruction, Optimization

\section{INTRODUCTION}

The experimental electromagnetic dosimetry of mobile phones has been very much developed recently. The relevant quantity to be measured is the Specifc Absorption Rate (SAR), which designates the electromagnetic power density deposited per unit mass of biological tissue. SAR measurements are required to assess the compliance of mobile phones with existing standards and recommendations. Most of the existing dosimetric facilities utilize automatic positioning systems to move an isotropic E-Field measuring probe in a head-like phantom [1] (Figure 1). The procedure to measure SAR is described in the European Standard prEN50361 [2], or the IEEE version [3]. Typically, no less than 300 measurement points in the phantom are prescribed for accurate SAR measurements. The electromagnetic properties of the liquid £lling the phantom are similar to those of biological tissues, and are expected to provide a worst case, when compared to heterogeneous models of the head. According to the current standard procedure, a complete phone test will lasts about half a day. Indeed, the SAR induced by GSM mobile phones must be tested for different con£gurations, namely several frequencies in each band $(900 \mathrm{MHz}$ and $1800 \mathrm{MHz}$ ) and two positions with respect to the head, and for right and left hand users. Consequently, the rapidity of SAR measurement constitutes a key issue. Several attempts to reduce SAR measurement time are based, for example, on the use of a priori knowledge of the £eld decay in the phantom, or on the observed correlation of the local peak SAR and the averaged SAR. In the £rst case, the number of measurement points can be reduced, the feld probing being limited to a single plane, instead in a volume as imposed by the standard [4]. In the second one, the averaged SAR can be deduced from a quick manual search of the maximum £eld distribution in the phantom [5].

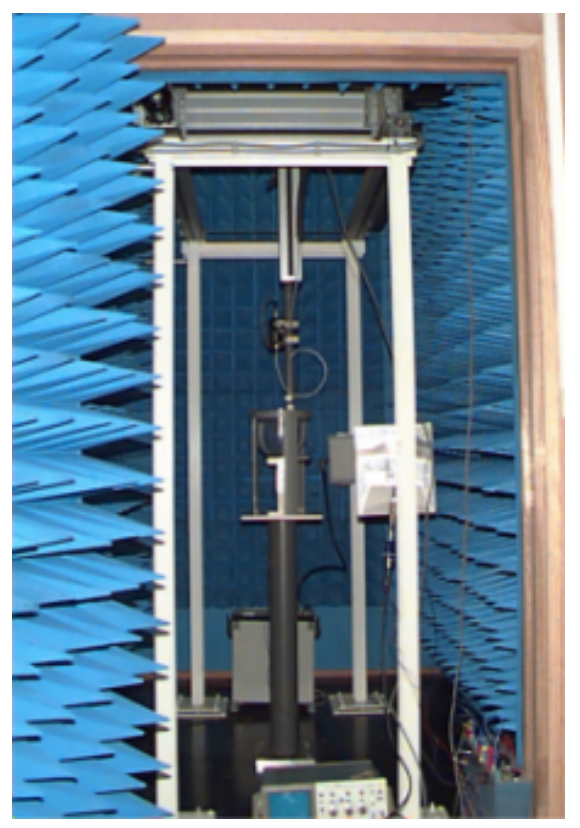

Fig. 1. Supélec dosimetric facility

This paper presents a new approach where SAR calculation is based on a parametric reconstruction of the E-£eld distribution in the phantom from a minimum number of sampling points. It has been shown that the £eld distribution in the phantom can be adequately described by means of an ellipsoidal model involving only 11 parameters. No more than 36 points are needed to determine these parameters from which the SAR 
can be accurately deduced. As a consequence, the acquisition time is reduced from at least $10 \mathrm{~min}$ to only $1 \mathrm{~min}$ per confguration. The local SAR is given by the following formula:

$$
S A R=\frac{\sigma E^{2}}{2 \rho} \quad\left(\mathrm{W} . \mathrm{kg}^{-1}\right)
$$

where $E(\mathrm{~V} / \mathrm{m})$ represents the electric £eld, $\sigma(\mathrm{S} / \mathrm{m})$ the conductivity and $\rho\left(\mathrm{kg} / \mathrm{m}^{3}\right)$ the volumic mass. The averaged SAR in $1 \mathrm{~g}$ and $10 \mathrm{~g}$ of contiguous tissues results from the volumetric integration of formula (1). In the last section, the averaged $1 \mathrm{~g}$ and $10 \mathrm{~g}$ SAR results obtained from measurements conducted on a large number of commercial phones, with 36 sampling points, validate the parametric approach.

\section{PHYSICAL CHARACTERISTICS OF THE E-FIELD}

\section{A. Decrease of the E-Field in function of depth}

The construction of the ellipsoidal model representing the E-feld distribution in a phantom is based on some physical assumptions. In a lossy homogeneous phantom, the decrease of the E-feld is necessarily monotonous and follows a priori an exponential decay in function of depth. This has been verifed with systematic SAR tests on a hundred of real mobile phones (at 2 frequencies $-900 \mathrm{MHz}$ and $1800 \mathrm{MHz}$ ). The curves corresponding to each tested phone (Fig. 1) are distributed around the line corresponding to the theoretical propagation constant $\alpha$ of the plane wave:

$$
E(x, y, z)=E(x, y, d) e^{-\alpha(z-d)}
$$

For the standard materials used, and for a plane wave, $\alpha=$ $-29.4 \mathrm{~Np} / \mathrm{m}$ at $900 \mathrm{MHz}$, and $\alpha=41.4 \mathrm{~Np} / \mathrm{m}$ at $1800 \mathrm{MHz}$.

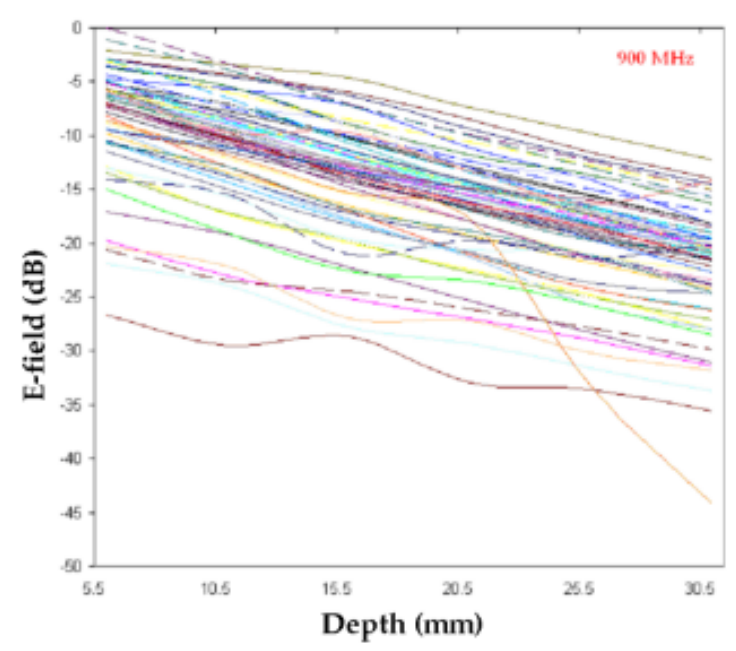

Fig. 2. Depth decrease of the maximum normalized E-Field, for 64 phones at $900 \mathrm{MHz}$.

\section{B. Attenuation and propagation}

In a homogeneous and isotropic media (inside the phantom), the E-feld can be represented as a plane wave sum. When the media is conductive, it is however necessary to take into account the introduction of the losses. Furthermore, the presence of evanescent waves has to be studied. The E-Field inside the phantom can be seen as the integration of the angular plane wave spectrum $\mathbf{A}(\mathbf{u})$ in the considered domain $\Omega$ :

$$
\mathbf{E}(\mathbf{r})=\iint_{\boldsymbol{\Omega}} \mathbf{A}(\mathbf{u}) \mathbf{e}^{-\mathbf{j k r} \cdot \mathbf{u}} \mathrm{d} \boldsymbol{\Omega}(\mathbf{u})
$$

with $\mathbf{u}=\{\alpha, \beta, \gamma\}, \alpha^{2}+\beta^{2}+\gamma^{2}=1$, and $\gamma=$ $\left(1-\left(\alpha^{2}+\beta^{2}\right)\right)^{-1}$.

Each plane wave can be represented with the following standard form, in which the term $k$, the propagation constant, is a unique complex scalar:

$$
\mathbf{E}(\mathbf{r})=\mathbf{E}_{\mathbf{0}}(\mathbf{r}) e^{-j k z}
$$

In function of the physical characteristics of the media $k^{2}=\omega^{2} \mu_{0} \varepsilon_{0} \varepsilon_{r}$, with $\varepsilon_{r}=\varepsilon_{r}^{\prime}-j \varepsilon_{r}^{\prime \prime}$, the complex propagation "constant" $k$ is expressed as follows,

$$
k=\frac{\omega}{c}\left(\sqrt{\frac{\left|\varepsilon_{r}\right|+\varepsilon_{r}^{\prime}}{2}}-j \sqrt{\frac{\left|\varepsilon_{r}\right|-\varepsilon_{r}^{\prime}}{2}}\right)
$$

with $k=k^{\prime}-j k^{\prime \prime}$. The plane wave is represented in the homogeneous conductive media with the equation:

$$
\mathbf{E}(\mathbf{r})=\mathbf{E}_{\mathbf{0}}(\mathbf{r}) e^{-j k^{\prime} z} e^{-k^{\prime \prime} z}
$$

The imaginary part of $k$ is responsible for the attenuation $\alpha$ of the wave, due to the losses in the equivalent liquid. We then introduce $\alpha=\Im m(k)$, expressed in Np.m ${ }^{1}$, which is the inverse of the penetration depth. $k^{\prime \prime}$ represents somewhat the skin thickness in the media. The real part $k^{\prime}$ of the wave vector allows defning the wavelength in this media. For a standard phantom and the plane wave, the values of $\alpha$ regarding the frequency are:

\begin{tabular}{|c|c|c|}
\hline Plane wave & $900 \mathrm{MHz}$ & $1800 \mathrm{MHz}$ \\
\hline$\alpha(\mathrm{Np} / \mathrm{m})$ & $-29,4$ & $-41,4$ \\
\hline
\end{tabular}

TABLE I

PLANE WAVE ATTENUATION IN A BIOLOGICAL MEDIA

When the phantom is exposed to a mobile phone, the E-£eld inside is of course not a plane wave but a linear combination of plane waves. As we will see, the value of $\alpha$ depends strongly of the frequency, but also of the phone under test. The accuracy of the measurement, directly related to the strength of the E-£eld in the phantom, plays also a role in the determination of $\alpha$. On the contrary, in order to describe the distribution of 
the E-£eld in a phantom with a simple model, to limit the number of parameters, it is necessary to fnd a convenient way of determination for a unique propagation constant $\alpha$, at least for each frequency band. The calculation of $\alpha$ from a few points distributed in function of depth on a line perpendicular to the planar phantom interface doesn't allow describing the exact behaviour of the electric £eld, but gives an interesting approach. This principle has still been applied to describe the decrease of the electric feld in a phantom for a rapid SAR measurement [6]. To improve the calculation, $\alpha$ should be dependent of the plane wave spectrum of the E-£eld.

As a £rst approximation, the value of $\alpha$ is calculated for each phone as the slope value of the lines observed in the £gure 3 . This fgure shows the values of $\alpha$ obtained from the maximum amplitude of the electric £eld, and from the averaging of all the propagation constants calculated for each point of the measurement surface. The horizontal line represents the corresponding plane wave propagation constant value, at $900 \mathrm{MHz}$ and $1800 \mathrm{MHz}$. The measurement is much less noisy in the $900 \mathrm{MHz}$ band than in the $1800 \mathrm{MHz}$ band, where the attenuation is stronger. The sensitivity of the E-£eld measuring probe (about $1 \mathrm{~V} / \mathrm{m}$ ) is unfortunately, for some phones, too low to give a good accuracy for the measurement.

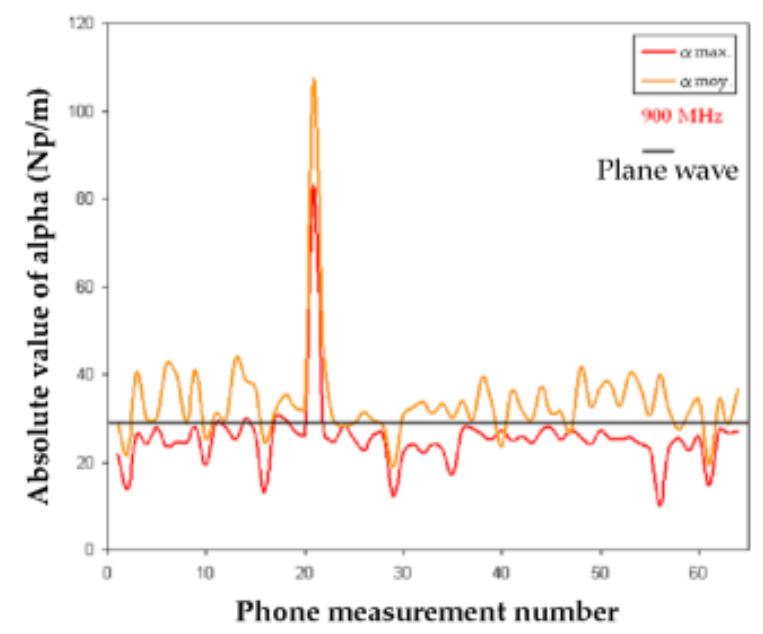

Fig. 3. Absolute values of the mean and maximum propagation "constant" $\alpha$ $\left(\mathrm{Np} . \mathrm{m}^{1}\right)$ at $900 \mathrm{MHz}$ for 64 phones

We have represented the distribution of the propagation constant in function of its values, by means of the histograms of the fgure 4. With the corresponding distribution functions, we then deduced the $10 \%$ and $90 \%$ quantiles. The $q$ order quantile is the inverse function for the distribution function. It gives the value $\alpha_{n}$ so that Prob $\left(\alpha \leq \alpha_{n}\right)=q$. It allows obtaining an estimation of the upper bound of $\alpha$ with a probability $q$ that this value will not be under-estimated.

The calculation of the parameter $\alpha$ gives a quasi gaussian distribution of the values at $900 \mathrm{MHz}$, that allows evaluating precisely, from the distribution function, the $10 \%$ and $90 \%$

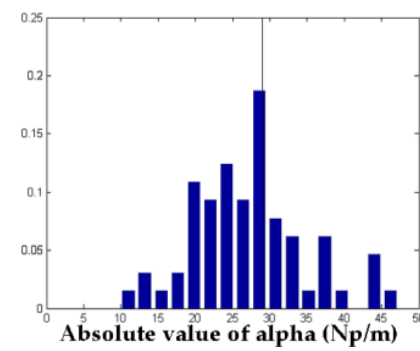

(a) histogram

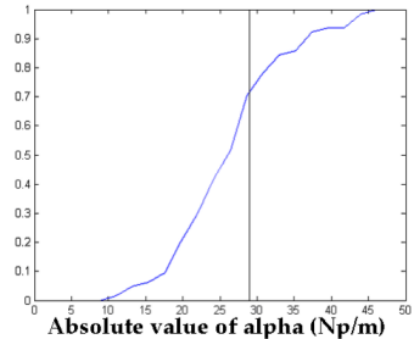

(b) distribution function
Fig. 4. Absolute values of the maximum propagation "constant" $\alpha\left(\mathrm{Np} \cdot \mathrm{m}^{1}\right)$ at $900 \mathrm{MHz}$ for 64 phones represented as a histogram (a) and its corresponding distribution function (b)

quantiles. At $1800 \mathrm{MHz}$, the distributions are more dif£cult to characterize in terms of statistical laws. At $900 \mathrm{MHz}$, determining this value is not dif£cult, the different values are very close, but at $1800 \mathrm{MHz}$ the measurement accuracy is poor, resulting in many different values for the propagation constant. The selected values of $\alpha$ representing the global decrease of the electric £eld in the phantom are equal to $23 \mathrm{~Np} / \mathrm{m}$ at $900 \mathrm{MHz}$ and $55 \mathrm{~Np} / \mathrm{m}$ at $1800 \mathrm{MHz}$. The use of the penetration depth as an E-£eld depth decrease have been also recently used to build a fast method of averaged SAR estimation [4].

\section{Geometrical E-£eld distribution}

The characteristics of the propagation medium have an inauence on the electromagnetic propagation, but also on the shape of the E-£eld. It is commonly accepted that the spatial distribution of the electric £eld is more or less of ellipsoidal type (or of "potatoe" shape, more exactly) for the large majority of the measurements done. With the benefts of an important data base of SAR and E-£eld measurements for about hundred commercial phones, we assume that the distribution of the E-£eld in the phantom is much more simple than it could appear. It can be observed, especially at $1800 \mathrm{MHz}$, but very occasionally, some distributions with double SAR spots, due to particular phones, like clam-shells for example. But even in this case, the double spot distribution can be separated in two ellipsoids. The fgure 5 shows an example of E-£eld shapes for typical commercial phones in the plane close to the bottom of the phantom (6 $\mathrm{mm}$ above it). The E-£eld data have been obtained with a meshing step of $7 \mathrm{~mm}$, in $70 \mathrm{~mm}$ size squares. These examples are characteristic of those met the most often in our own data base.

\section{DEGREES OF FREEDOM}

The elliptic spatial distribution of the E-£eld and its propagation as an exponential function of depth are characteristics 


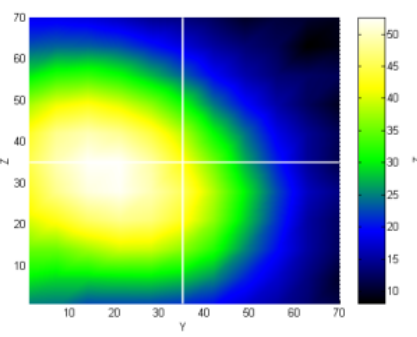

(a)

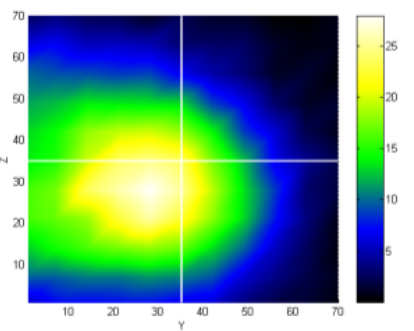

(b)
Fig. 5. Classical E-£eld distribution in a plane normal to the surface phantom (a) and in function of depth (b)

relatively easy to model. These investigations let think that giving morphological and physical considerations on the E-feld must allow introducing a priori information in the SAR calculation [7]. The number of data points required in the standard way to compute the SAR should be decreased, by considering that a large number of those points depend from each other from electromagnetic or morphological laws. The electric feld in the phantom can then be seen, in that way, as a relatively simple system: the E-£eld generated by a spherical wave expansion, as a £rst approximation. A £rst estimation of the degree of freedom $N$ of the distribution of the electric £eld in the phantom can be evaluated by the number of useful terms of its development in spherical modes [8]: $N=\lfloor k r\rfloor+10$, where $k=\frac{2 \pi f}{v_{\phi}}$ and where the square brackets indicate the largest integer smaller than or equal to $k r, r$ is the radius of the domain of study i.e. the sphere of smallest radius that circumscribes the system, $v_{\phi}$ the velocity of light, $f$ the frequency in the liquid. As an example, for $r=3.5 \mathrm{~cm}$ we obtain $N \approx 14$ for $f=900 \mathrm{MHz}$, and $N \approx 18$ for $f=1800 \mathrm{MHz}$. We can then think that about 10 parameters of a mathematical model should be enough to reconstruct the E-£eld in the entire volume from a reduced number of data points.

\section{PROPOSED APPROACH}

It has been observed, for a large number of mobile phones, that the spatial E-£eld distribution, in the phantom, looks like an elliptical "spot" or "potato", as shown on Figure 2. This has led to consider an ellipsoidal model to represent such £eld pro£les:

$$
E(\mathbf{p}, \mathbf{r})=E_{0} e^{-k(1-\rho) \sqrt{\left(x-x_{e}\right)^{2}+\left(y-y_{e}\right)^{2}+\left(z-z_{e}\right)^{2}}}
$$

where $\rho=\left(\frac{x^{\prime 2}}{a^{2}}+\frac{y^{\prime 2}}{b^{2}}+\frac{z^{\prime 2}}{c^{2}}\right)^{\frac{-1}{2}}$ and $\mathbf{r}^{\prime}=\mathbf{R}_{\alpha} \mathbf{R}_{\beta} \mathbf{R}_{\gamma} \mathbf{r}$ with $\mathbf{R}_{\alpha}, \mathbf{R}_{\beta}, \mathbf{R}_{\gamma}$, the rotation matrices built from the Euler rotation angles $\alpha, \beta, \gamma \mathbf{g}$, and $\mathbf{r}(x, y, z)$ the vector of positions of the Efeld measurement points. $\mathbf{p}\left(E_{0}, k, x_{e}, y_{e}, z_{e}, a, b, c, \alpha, \beta, \gamma\right)$ is the vector of parameters, where $E_{0}$ represents the E-£eld amplitude, $k$ is similar to the propagation term, $x_{e}, y_{e}$ and $z_{e}$ are the coordinates of the center of the ellipsoid, and $a, b$ and $c$ are the semi-axes of the ellipsoid.

From the $n$ measurement points chosen, the parameters $\mathbf{p}$ of the considered model are estimated in the sense of the least squares of the error $\xi(\mathbf{p})$ between the model $E(\mathbf{p}, \mathbf{r})$ and the measurement points $f(\mathbf{r})$ :

$$
\xi(\mathbf{p}, \mathbf{r})=\|f(\mathbf{r})-E(\mathbf{p}, \mathbf{r})\|^{2}
$$

The estimation procedure of the ellipsoidal parameters is a classical optimization resolution. The least-squares problem can be solved by using, for example, a Levenberg-Marquardt algorithm. In the estimation process, the parameters are normalized, in order to eliminate the scaling factors and then avoid the prevalence of some parameters on the others. When the parameters have been determined, the calculation of E-£eld at any point in the phantom is straightforward, and the computation of the maximum SAR integrated in 1 or $10 \mathrm{~g}$ can be performed. The choice of the measurement points in the initial mesh governs the correct estimation of the parameters. Extensive trials have shown that a good compromise consists both in one area scan in a plane close to the phantom interface and in 4 zoom scans in deeper planes (Fig. 3). The area scan allows localizing the maximum E-£eld area, while the zoom scans are expected to provide an information on the feld decay. The area scan consists of $4 \times 4$ sampling points. The zoom scans are centered on the maximum E-£eld area and consist of only 2x2 sampling points. Globally 36 sampling points are required to determine the 11 model parameters. The depth of the frst measurement plane (6 $\mathrm{mm}$ from the bottom of the phantom) is £xed for compromising suf£cient sensitivity and low probe interaction with the phantom wall

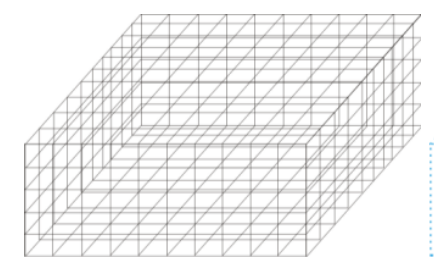

(a)

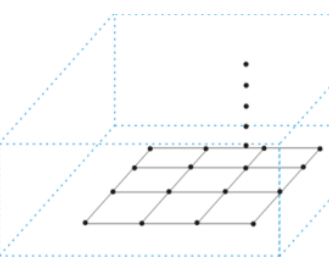

(b)
Fig. 6. standard cubic meshing of 300 E-£eld points (a), and fast SAR 36 E-£eld points meshing (b)

The £rst planar meshing, relatively close to the radiation source, allows to localize the maximum E-£eld zone, in what the maximum averaged SAR cube will necessary be, whereas the depth exploration in this area gives information on the decrease of the feld. This crucial information is required for the correct estimation of the parameters. The depth of the £rst plane ( $6 \mathrm{~mm}$ from the bottom of the phantom) is £xed for setting suf£cient sensitivity and low interaction with the phantom wall. 


\section{RESULTS}

The parametric reconstruction technique has been assessed from 128 measurements conducted on 64 dual-band commercial GSM mobile phones, at $900 \mathrm{MHz}$ and $1800 \mathrm{MHz}$. the following fgures show the correlation between 1) the maximum SAR averaged on $10 \mathrm{~g}$ and $1 \mathrm{~g}$ obtained by the parametric reconstruction, from 36 measurement points, and 2) the same quantity deduced from the standard procedure involving 300 measurement points. The £gure 7 presents the results obtained with 16 measured data points plus 5 extrapolated points calculated with the plane wave propagation constant $\alpha$, while fgure 8 show results obtained with 16 measured data points plus 5 extrapolated points calculated with the mean propagation value $\alpha$. Those results are compared to the standards measurements obtained from 726 E-£eld data points. The accuracy of the method (the mean error is $10 \%$ for the 21 measured data points) has to be compared with the complete error made with standard dosimetric facilities, which is commonly $30 \%$ to $40 \%$. The reproducibility between two measurements for the same phone and with the same assessment is about $5 \%$ to $10 \%$ - The choice of an E-£eld propagation model based on real measurements is therefore better than the simplest description of the plane wave. This result confrm the previous observation of the relatively random decreases of the E-£eld as a function of depth. However, the approach using the plane wave propagation constant, modifed by the use of lower propagation constants ensuring a maximized value for the calculated SAR, could nevertheless £nd an application in very fast measurements, for pre-compliance or systematic production tests for example.

Figure 9 shows SAR results obtained from 36 measurement points, near the surface and in depth of the phantom, without the use of the propagation constant. In this case, $\alpha$ is not explicitly used, but the measurements in depth give the information of the E-£eld decrease, for each phone measured. This is the only way to precisely match $\alpha$ for each case. The regression curve superposed to the measurement points shows the good accuracy of the parametric reconstruction approach. The mean relative error calculated is about $5 \%$, to be compared to the $30 \%$ of uncertainty commonly observed for the standard measurement procedure. This con£guration of 36 points is optimum to obtain a good accuracy and a fast measurement. Using only 21 measured points for example leads to a degradation of the accuracy to about $10 \%$.

The effciency of the parametric reconstruction technique can be explained by the similarity existing between the actual feld distribution and the model for most of the commercial phones, and by the fact that the averaged SAR involves an integration of this £eld distribution. On the contrary, more surprising is that the parametric reconstruction proved also very ef£cient, even in the case of multi-spot E-£eld distributions which are sometimes encountered for "đip-øop" phones and/or in the upper GSM frequency band. An explanation is that, in

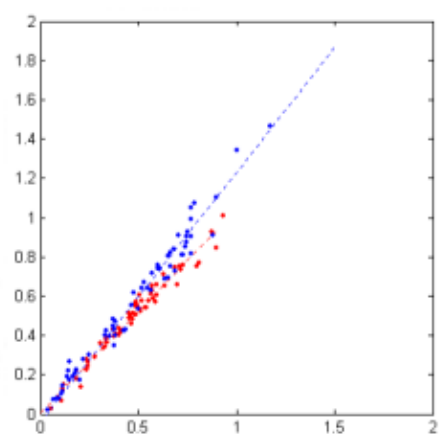

(a)

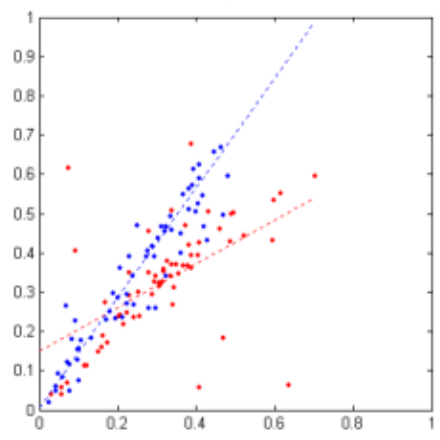

(b)

Fig. 7. Reconstructed SAR (W/kg) in function of the standard SAR, integrated in $1 \mathrm{~g}$ (a) and in $10 \mathrm{~g}$ (b), with $16 \mathrm{E}$-£eld points and 5 points calculated with the plane wave propagation constant.

such cases, the zoom scans are centered on the maximum spot area, which is predominantly responsible of the SAR value.

\section{CONCLUSIONS}

This parametric approach applied to SAR measurement domain has already proved its effciency with the robust estimation of other physical quantities [9]. The method proposed in this paper allows reducing the number of sampling points from 300 , for the standard approach, to only 36 . The error made on the $10 \mathrm{~g}$ or $1 \mathrm{~g}$ averaged SAR with this interpolation technique do not contribute much than a few percent to the global uncertainty of the measurement method. The acquisition time for a given confguration is about 1 minute $(36 \mathrm{E}$ feld points), including data processing, which is very fast. The method is fully compatible with popular instrumentation and, hence, can be directly implemented as option on most existing SAR measurement facilities using mechanical scanning of an E-feld probe [10], [11]. Furthermore, the hybrid ellipsoidalpropagation constant approach, used in conjunction with an array of E-£eld probes, should be able to provide quasi real time 


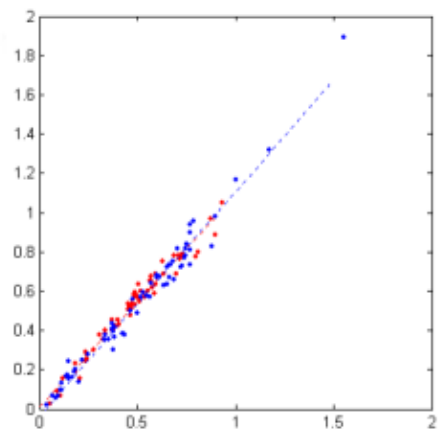

(a)

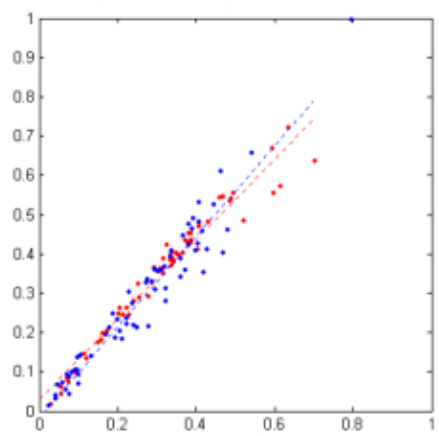

(b)

Fig. 8. Reconstructed SAR (W/kg) in function of the standard SAR, integrated in $1 \mathrm{~g}$ (a) and in $10 \mathrm{~g}$ (b), with $16 \mathrm{E}$-£eld points and 5 points calculated with the mean propagation constant.

SAR measurement capabilities.

\section{References}

[1] T. Schmid, O.Egger, N. Kuster, "Automated E-Field scanning system for dosimetric assessments," IEEE Trans. MTT, vol. 44, no. 1, pp. 105-113, Jan. 1996.

[2] CENELEC TC211, "Basic standard for the measurement of Specifc Absorption Rate related to human exposure to electromagnetic felds from mobile phones (300 MHz - 3 GHz)," PrEN 50361 European Standard, Jun. 2000.

[3] IEEE 1528 Standard "Recommended Practice for Determining the Peak Spatial-Averaged Specifc Absorption Rate (SAR)in the Human Body Due to Wireless Communication Devices: Experimental Techniques,' IEEE SCC34 SC2 Standard 1528, Jun. 2003.

[4] M. Y. Kanda, M. Ballen, M. G. Douglas, A. Gessner, and C-K. Chou, "Fast SAR determination of gram-averaged SAR from 2-D coarse scans," Proc. 25th Ann. Meeting of the Bioelectromagnetics Soc. (BEMS), pp. 45-46, Wailea, Maui, USA, Jun. 2003.

[5] Indexsar, MapSAR system, www.indexsar.com..

[6] M. Manning, P. Massey, "FRapid SAR Testing of Mobile Phone Prototype Using a Spherical Test Geometry," Proc. IEE Technical Seminar on Antenna Measurements and SAR (AMS 2002), Loughborough University, UK, May. 2002.

[7] L. Sandu, J. Oksman, G. Fleury, "Information criteria for the choice of parametric functions for measurement," IEEE Transactions on Measurement, vol. 47, n4, pp. 920-924, 1998.

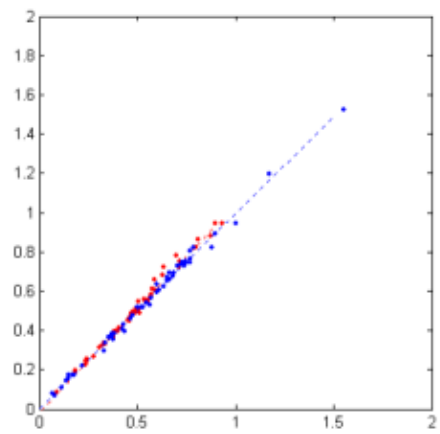

(a)

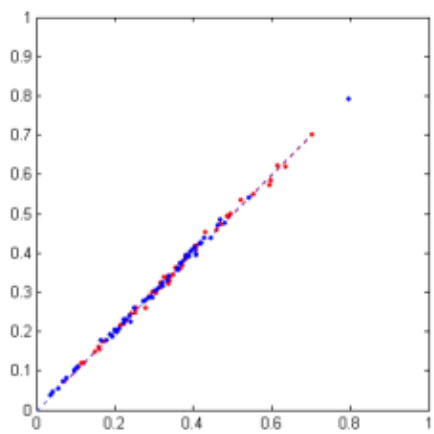

(b)

Fig. 9. Maximum SAR averaged on $10 \mathrm{~g}$ (a) and $1 \mathrm{~g}$ (b), for the standard measured values (abscissa), and the values obtained from reconstructions with 36 points (ordinate), at 900 and $1800 \mathrm{MHz}$.

[8] J. E. Hansen, J. Oksman, G. Fleury, "Spherical near-£eld antenna measurements," IEE Electromagnetic Waves Series , n26, 1988.

[9] G. Fleury, M.-E. Davoust, "Remote Field Eddy Current Inspection for Groove Sizing Choice of a Direct Model Structure," Review of Progress in Quantitative Nondestructive Evaluation - American Institute of Physics, vol. 19A, n 509, pp. 541-548, 1999.

[10] O. Merckel, G. Fleury, J.-Ch. Bolomey, "Propagation model choice for rapid SAR measurement," Proc. XIth European Signal Processing Conference (EUSIPCO 2002), pp. 80, Toulouse, France, Sept. 2002.

[11] O. Merckel, J.-Ch. Bolomey, G. Fleury, "Extension of the parametric rapid SAR measurement to the SAM phantom," Proc. 6th International Congress of the European Bioelectromagnetics Association (EBEA 2003), Budapest, Hungary, Nov. 2003. 